\title{
Suspension syndrome: a potentially fatal vagally mediated circulatory collapse-an experimental randomized crossover trial
}

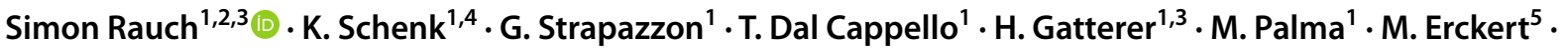 \\ L. Oberhuber $^{6} \cdot$ B. Bliemsrieder $^{7} \cdot$ H. Brugger ${ }^{1} \cdot$ P. Paal $^{8}$
}

Received: 25 October 2018 / Accepted: 13 March 2019 / Published online: 20 March 2019

(c) The Author(s) 2019

\begin{abstract}
Purpose Suspension syndrome describes a potentially life-threatening event during passive suspension on a rope. The pathophysiological mechanism is not fully understood and optimal treatment unknown. We aimed to elucidate the pathophysiology and to give treatment recommendations.

Methods In this experimental, randomized crossover trial, 20 healthy volunteers were suspended in a sit harness for a maximum of $60 \mathrm{~min}$, with and without prior climbing. Venous pooling was assessed by measuring the diameter of the superficial femoral vein (SFV), lower leg tissue oxygenation $\left(\mathrm{StO}_{2}\right)$ and by determining localized bioelectrical impedance. Hemodynamic response was assessed by measuring heart rate, blood pressure, stroke volume, and left ventricular diameters. Signs and symptoms of pre-syncope were recorded.

Results Twelve (30\%) out of 40 tests were prematurely terminated due to pre-syncopal symptoms (mean $44.7 \mathrm{~min}$, minimum 13.4, maximum 59.7). SFV diameter increased, $\mathrm{StO}_{2}$ and the capacitive resistance of the cells decreased indicating venous pooling. Heart rate and blood pressure did not change in participants without pre-syncope. In contrast, in participants experiencing pre-syncope, heart rate and blood pressure dropped immediately before the event. All symptoms dissolved and values returned to normal within 5 min with participants in a supine position.

Conclusions Sudden pre-syncope during passive suspension in a harness was observed in $30 \%$ of the tests. Blood pools in the veins of the lower legs; however, a vagal mechanism finally leads to loss of consciousness. Time to pre-syncope is unpredictable and persons suspended on a rope should be rescued and put into a supine position as soon as possible.
\end{abstract}

Keywords Suspension syndrome $\cdot$ Harness hang syndrome $\cdot$ Suspension trauma $\cdot$ Climbing $\cdot$ Pathophysiology $\cdot$ Treatment

Communicated by Massimo Pagani.

Electronic supplementary material The online version of this article (https://doi.org/10.1007/s00421-019-04126-5) contains supplementary material, which is available to authorized users.

Simon Rauch

simon.rauch@eurac.edu

1 Institute of Mountain Emergency Medicine, Eurac Research, Viale Druso 1, 39100 Bolzano, Italy

2 Department of Sports Science, Medical Section, University of Innsbruck, 6020 Innsbruck, Austria

3 Department of Anesthesiology, University Hospital LMU Munich, 80337 Munich, Germany

4 Department of Sports Medicine, Pro Motus, 39100 Bolzano, Italy

\begin{tabular}{|c|c|}
\hline \multicolumn{2}{|c|}{ Abbreviations } \\
\hline BIA & Body impedance analysis \\
\hline $\mathrm{BP}$ & Blood pressure \\
\hline HR & Heart rate \\
\hline LMM & Linear mixed model \\
\hline LVEDD & Left ventricular end-diastolic diameter \\
\hline LVESD & Left ventricular end-systolic diameter \\
\hline
\end{tabular}

5 Department of Cardiology, F. Tappeiner Hospital, 39012 Merano, Italy

6 Department of Internal Medicine I, Gastroenterology, Hepatology, Metabolism and Endocrinology, Medical University Innsbruck, 6020 Innsbruck, Austria

7 Department of Anesthesiology, Garmisch-Partenkirchen Medical Center, 82467 Garmisch-Partenkirchen, Germany

8 Department of Anesthesiology and Intensive Care Medicine, Brothers of St. John of God Hospital, Paracelsus Medical University, Kajetanerplatz 1, 5010 Salzburg, Austria 


$\begin{array}{ll}H & \text { Height } \\ \text { NIRS } & \text { Near-infrared spectroscopy } \\ R & \text { Resistance } \\ \text { SBP } & \text { Systolic blood pressure } \\ \mathrm{SFV} & \text { Superficial femoral vein } \\ \mathrm{SV} & \text { Stroke volume } \\ \mathrm{ScO}_{2} & \text { Cerebral oxygen saturation } \\ \mathrm{StO}_{2} & \text { Tissue oxygen saturation } \\ \mathrm{Xc} & \text { Reactance }\end{array}$

\section{Introduction}

Suspension syndrome (also called suspension trauma) describes a potentially life-threatening event induced by passive hanging on a rope or in a harness system in a vertical or near-vertical position (Flora 1972b; Pasquier et al. 2011; Roggla et al. 2008). Suspension syndrome does not only play a role in various occupational activities where harness systems are used, such as inspection, painting and construction on high-rise buildings, large facades, pylons, bridges, dams, off-shore platforms and power plants, but also in sports such as rock climbing, ice climbing, mountaineering, canyoning and caving (Pasquier et al. 2011). The first case series of suspension syndrome in climbers was reported in 1972: 10 out of 23 climbers who had fallen in the rope died after hanging in their harness, though they did not suffer any traumatic injuries (Flora 1972b). Since then, the pathophysiology of the suspension syndrome has been debated controversially (Lee and Porter 2007). The most widespread hypothesis assumes blood pooling in the lower limbs, prompting a reduction in cardiac preload and subsequently a decrease in cardiac output and tissue perfusion, eventually leading to loss of consciousness and cardiac arrest (Lee and Porter 2007). However, no study has ever proven this theory, and alternative pathophysiological mechanisms have been discussed (Halliwill et al. 2014; Roeggla et al. 1996). Specifically, a vagal mechanism leading to sudden bradycardia and hypotension has been hypothesized.

Since the first descriptions of suspension syndrome, the immediate aid by first responders has been debated (Thomassen et al. 2009). Some current recommendations still advise against placing a casualty in a supine position after being rescued from suspension and recommend a semirecumbent position (Weems and Bishop 2003). The basis for this recommendation is the hypothesis that blood returning from the legs upon horizontal positioning would lead to an acute volume overload of the right heart and eventually cause rescue death. However, this hypothesis is based on expert opinion and case reports presented in 1972 (Flora 1972a) and has never been proven.

The main aim of our study was to elucidate the pathophysiological mechanism leading to suspension syndrome and to derive recommendations for prevention and treatment. In particular, we wanted to differentiate between a relevant reduction in cardiac preload due to venous pooling, and a vagally mediated event. In addition, we hypothesized that passive hanging in a harness with prior physical activity would increase the susceptibility and decrease the time to the occurrence of suspension syndrome, particularly if a vagal mechanism was involved. In fact, postexercise syncope, although incompletely understood, is a known entity (Lowe and Petch 2000; O'Connor et al. 1999; Shen et al. 2017), and in real scenario, a certain amount of exercise usually precedes the fall into the rope.

\section{Methods}

\section{Study approval and ethical background}

This experimental randomized crossover study was approved by the Institutional Review Board of the General Hospital of Bolzano, Italy (no. 68-2015) and registered in ClinicalTrials.gov (trial number NCT02726776). The study was conducted according to the Declaration of Helsinki (World Medical Association Declaration of Helsinki: ethical principles for medical research involving human subjects 2013), Convention on Human Rights and Biomedicine (Convention for Protection of Human Rights and Dignity of the Human Being with Regard to the Application of Biology and Biomedicine: Convention of Human Rights and Biomedicine 1997) and the regulations of the Council for International Organizations of Medical Sciences (International ethical guidelines for biomedical research involving human subjects 2002). Written informed consent was obtained from all participants before enrolment.

\section{Study population}

The study was performed on 20 healthy [American Society of Anesthesiologists (ASA) classification class 1], male, non-professional climbers (mean age 31.1, range 21-46 years).

Before enrolment, a medical history, physical examination and a 12-lead ECG were obtained and volunteers with pre-existing illnesses (ASA-class $>1$ ) or any abnormal finding in the physical examination or the ECG were excluded. Participants did not take any medications and did not have a history of recurrent syncopal events. In an echocardiographic exam prior to the study, cardiopmyopathies or significant valvular pathologies were excluded. Participants were asked to avoid moderate and intense physical activity 
as well as alcohol and coffee consumption in the $24 \mathrm{~h}$ preceding the study.

\section{Study design, setting, and instrumentation}

The study design is depicted in Fig. 1. The study was performed in an indoor climbing facility. In the "pre-suspension phase", baseline values were obtained in a supine position (after $5 \mathrm{~min}$ of rest) and in a standing position ( 2 min after standing up) for the diameter of the right-sided superficial femoral vein (SFV), tissue oxygen saturation $\left(\mathrm{StO}_{2}\right)$ of the right calf muscle, bioelectrical-impedance patterns of the lower leg (localized bioelectrical-impedance), heart rate (HR), blood pressure (BP), left ventricular end-systolic and end-diastolic diameter (LVESD and LVEDD) and cerebral oxygen saturation $\left(\mathrm{ScO}_{2}\right)$. The diameter of the right $\mathrm{SFV}$ was measured with a linear, 5-10 MHz ultrasound probe (DP50, Mindray Bio-Medical Electronics Co., Ltd., Shenzhen, China). $\mathrm{StO}_{2}$ of the calf muscle was measured using nearinfrared spectroscopy (NIRS) (O3 ${ }^{\mathrm{TM}}$ Regional Oximetry, Masimo Corporation, Irvine, USA) with the probe placed posterolaterally at the level of maximal calf circumference. Single-frequency $(50 \mathrm{kHz})$, tetrapolar, localized bioelectrical-impedance measurements were performed using the fibular head and the sinus tarsi as landmarks for the placement of the proximal and distal pair of electrodes, with the current driving and voltage sensing electrodes placed one next to the other (BIA 101 Anniversary device, Akern, Florence, Italy, phase sensitive BIA device). From phase sensitive bioelectrical-impedance analysis, resistance $(R)$ and reactance $(\mathrm{Xc})$ values can be derived. $R$ is the opposition to the flow of an alternating current and is inversely related to fluid volume, whereas Xc indicates the capacitive component of the cell membranes (Lukaski 2013; Nescolarde et al. 2015). Thus, localized bioelectrical-impedance may be used to identify regional fluid accumulation and cell membrane integrity and function (Nescolarde et al. 2015). Additionally, localized bioelectrical-impedance has been related to blood circulation properties (Varlet-Marie et al. 2016). HR, $\mathrm{BP}$ and stroke volume (SV) were recorded non-invasively and continuously (beat-by-beat) (Nexfin ${ }^{\circledR} \mathrm{HD}, \mathrm{BMEYE} \mathrm{B.V \text {, }}$ Amsterdam, Netherlands) (Mehta and Arora 2014). From the inter-beat interval and the systolic BP recorded by the device, baroreceptor-sensitivity was calculated. The LVESD and LVEDD were determined in an apical four-chamber view using a 2.5-5 MHz probe (M5, Mindray Bio-Medical Electronics Co., Ltd., Shenzhen, China). $\mathrm{ScO}_{2}$ was measured using NIRS with the probe placed on the right-sided forehead.

Following baseline measurements, each participant was randomized into one of two groups: participants randomized into group 1 were asked to climb for $10 \mathrm{~min}$ at a moderate intensity before proceeding directly to the "suspension phase" without any discontinuity. Participants assigned to group 2 proceeded to the suspension phase without prior climbing. Adopting a crossover design, on a second study day, the participants switched the group (with a rest period of at least $48 \mathrm{~h}$ between the two tests). While climbing, participants were belayed in a sit harness according to the latest recommendations of the Austrian Alpine Club (Mössmer 2013).

During the "suspension phase", the participants were freely suspended on a rope in a sit harness (Rock M, Salewa, Oberalp SPA, Bolzano, Italy, appropriate size selected

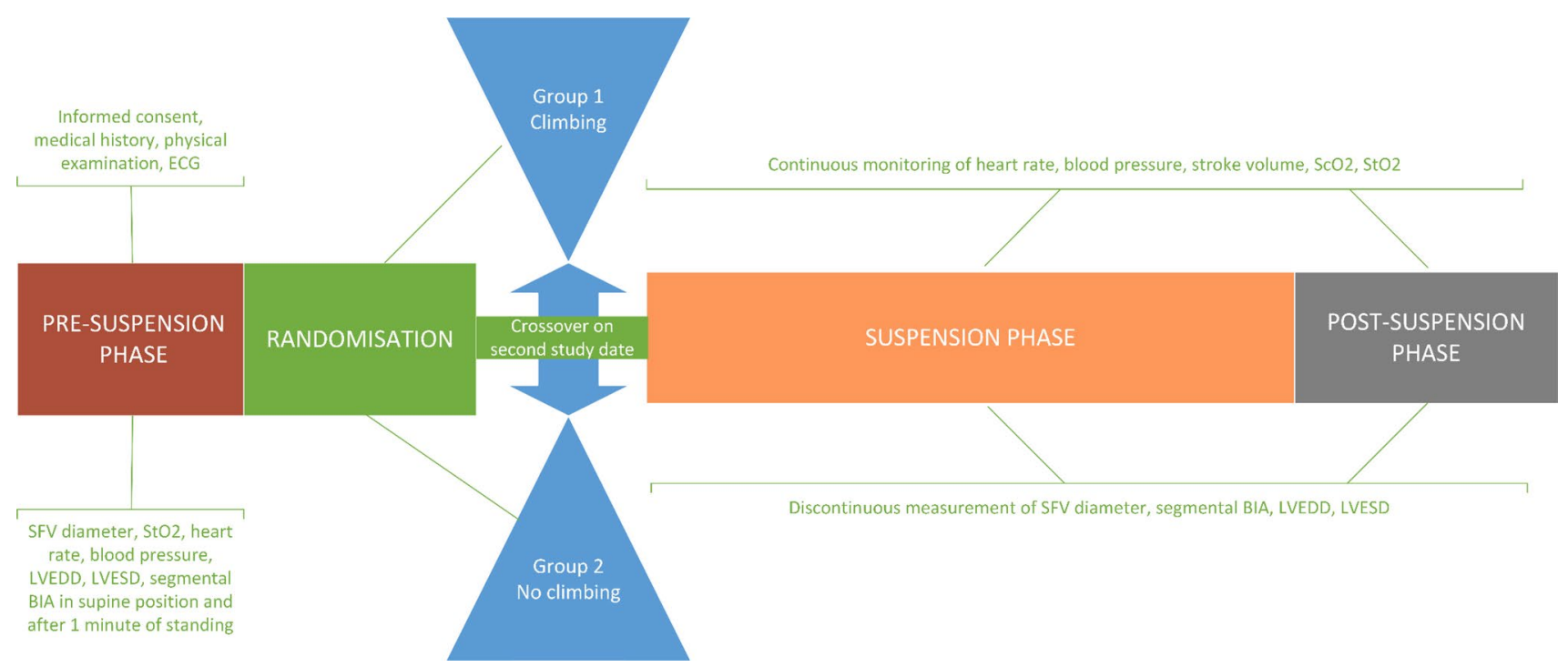

Fig. 1 Study design. $\mathrm{ScO}_{2}$ cerebral oxygen saturation, $\mathrm{StO}_{2}$ tissue oxygen saturation of the calf muscles, $L V E D D$ left ventricular end-diastolic diameter, $L V E S D$ left ventricular end-systolic diameter, $S F V$ superficial femoral vein, BIA body impedance analysis 
according to manufacturer's recommendation) about $50 \mathrm{~cm}$ above the ground. $\mathrm{HR}, \mathrm{BP}$ and $\mathrm{SV}$, as well as $\mathrm{ScO}_{2}$ and $\mathrm{StO}_{2}$ of the calf muscle were continuously monitored. The diameter of the SFV and the LVESD and LVEDD was determined every $2.5 \mathrm{~min}$ during the first $15 \mathrm{~min}$ of the suspension phase and in $5 \mathrm{~min}$ interval thereafter. Changes in localized bioelectrical-impedance were determined every $5 \mathrm{~min}$. Clinical signs and symptoms were assessed continuously and dizziness, light-headedness, pallor, warmth, blurred vision and nausea were considered signs and symptoms of a pre-syncope. Participants were asked to report the maximum level of pain during the suspension phase on a 10-step numeric rating scale (NRS) (Breivik et al. 2008).

The suspension phase was interrupted if one of the interruption criteria listed in Table 1 was met.

Participants were then immediately brought from the hanging position in a supine position ("post-suspension phase"). For another $15 \mathrm{~min}$, the participants' diameter of the $\mathrm{SFV}, \mathrm{StO}_{2}$, localized bioelectrical impedance, $\mathrm{HR}, \mathrm{BP}$, SV, LVESD and LVEDD as well as $\mathrm{ScO}_{2}$ were monitored.

\section{Data analysis}

Beat-to-beat data of HR, systolic BP and SV were first interpolated at $4 \mathrm{~Hz}$ (i.e., one value every $0.25 \mathrm{~s}$ was calculated) and then smoothed by means of a simple moving average of $30 \mathrm{~s}$ to reduce variability (for an example see Supplementary Figure 1). For the diameter of the $\mathrm{SFV}, \mathrm{StO}_{2}, \mathrm{HR}$, systolic BP, SV, LVEDD, LVESD and $\mathrm{ScO}_{2}$, a linear mixed model (LMM) was used to detect whether time, climbing, pre-syncope, pain ( $\leq 3$ and $>3$ on the NRS) and interactions of time with climbing, time with pre-syncope and time with pain had an effect during the suspension and post-suspension phases. For the LMM, different time points were defined: start of the suspension phase, $3 \mathrm{~min}$ before end of the suspension phase (when the most obvious changes of the parameters were noted), start of post-suspension phase and 5 min into the post-suspension phase. For SV, also 1 min before the end of the suspension phase was considered. Likewise, for the diameter of the SFV and $\mathrm{StO}_{2}$, an additional time point (minute 5 of the suspension phase) was used. The covariance

Table 1 Criteria for interruption of the suspension phase

Participant wishes to interrupt the study

Predefined maximal duration of $60 \mathrm{~min}$ reached

Heart rate $<35$ or $>160 \mathrm{bpm}$

Systolic blood pressure $<90 \mathrm{mmHg}$ or $>200 \mathrm{mmHg}$

Glasgow coma scale $\leq 13$

Decrease of cerebral oxygen saturation $>25 \%$ from baseline

Pre-syncopal signs and symptoms, i.e., dizziness, light-headedness, pale skin, a cold and clammy sweat, blurred vision and nausea

Bpm beats per minute structure for the residuals of the LMM was chosen by means of the Schwarz's Bayesian Criterion (BIC) among diagonal, compound symmetry, unstructured and first-order autoregression (AR(1)).

Baroreceptor-sensitivity was calculated as the ratio of the standard deviation of the inter-beat interval to the standard deviation of systolic BP, in accordance to previously proposed methods (Bernardi et al. 2010). Calculation was made every $5 \mathrm{~s}$ on a window size of $60 \mathrm{~s}$. Baroreceptor-sensitivity was calculated for two time intervals: minutes $5-10$ of the suspension phase (the first $5 \mathrm{~min}$ were not evaluated to have a more steady state) and the last minute of the suspension phase. The mean of the values of each interval was then calculated.

To compare two time points of a parameter as well as the means of the two intervals of the baroreceptor-sensitivity, the difference between them was used as dependent variable of a general linear model with participant as a random factor and climbing and pre-syncope as fixed factors. For multiple comparisons, the $P$ values were then corrected by means of Holm-Bonferroni method; according to this method, as soon as a null hypothesis is not rejected, all subsequent null hypotheses in the procedure are considered as non-significant, and therefore their $P$ value is denoted as $P=$ n.s. in the text. In the climbing and non-climbing sessions, Student's $t$ test was used to compare mean values of a parameter. Test durations of participants with pre-syncope in both climbing and non-climbing sessions were compared by means of Wilcoxon signed-rank test.

SPSS version 24.0 (IBM Corp., Armonk, NY, USA) was used for the statistical analysis, while the interpolation and the smoothing of HR, systolic BP and SV as well as the calculations of the baroreceptor-sensitivity were realized in $\mathrm{R}$ version 3.4.1. (R Development Core Team 2015). Values are reported as mean $\pm \mathrm{SD}$ and $P<0.05$ was considered statistically significant. Unless stated otherwise, the mean values refer to the 40 test samples, i.e., with and without climbing prior to the suspension phase.

\section{Results}

\section{Incidence, timing of pre-syncope, and the influence of climbing}

Twelve (30\%) out of the 40 tests were prematurely interrupted due to signs and symptoms of a pre-syncope, i.e., dizziness, light-headedness, pallor, warmth, blurred vision, and nausea (Fig. 2). The mean suspension time until the occurrence of the pre-syncope was $44.7 \pm 13.3 \mathrm{~min}$ (minimum 13.4, maximum 59.7). Seven of the $12(58.3 \%)$ pre-syncopal episodes occurred in participants who climbed before the suspension phase with a mean time to pre-syncope 


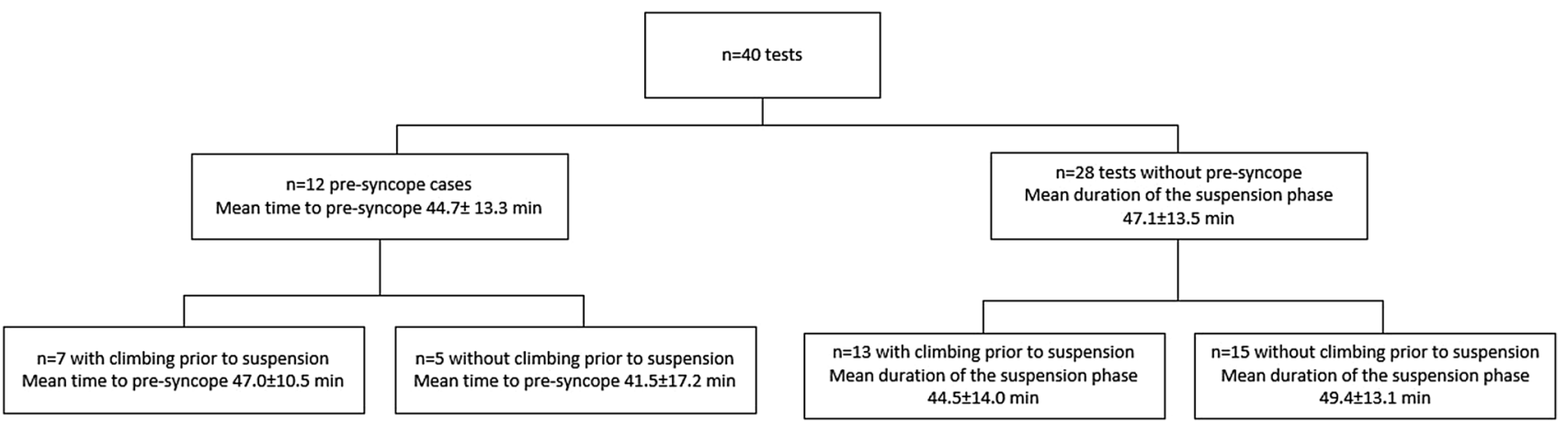

Fig. 2 Cases with and without pre-syncope, divided in climbing and no-climbing prior to the suspension phase

of $47.0 \pm 10.5 \mathrm{~min}$. The remaining five $(41.7 \%)$ pre-syncopal episodes were observed without climbing prior to the suspension phase and occurred after a mean time of $41.5 \pm 17.2 \mathrm{~min}$. Four participants experienced pre-syncope during both tests (i.e., with and without prior climbing). The time until pre-syncope of these four participants was $40.4 \pm 8.5 \mathrm{~min}$ with and $48.5 \pm 8.1 \mathrm{~min}$ without climbing $(P=0.068)$.

The 28 tests without a pre-syncopal episode were interrupted because the predefined maximal duration of the suspension phase $(60 \mathrm{~min})$ was reached $(43 \%)$ or due to pain (36\%) or numbness/palsy (21\%) in the lower extremities. The mean duration of the suspension phase in tests without a pre-syncope was $47.1 \pm 13.5 \mathrm{~min}$.

\section{Pain, numbness, and palsy in the lower extremities}

During 24 tests $(60 \%)$, participants reported pain in the lower extremities $\geq 3 / 10$ on a 10 -step NRS (Breivik et al. 2008). Among participants who experienced a pre-syncope, $75 \%$ ( 9 out of 12) reported a pain level $\geq 3$ on the NRS.
Although pain level did not differ between participants with and without a pre-syncope neither in the climbing session $(5.1 \pm 2.9$ vs. $4.0 \pm 2.8$ on the NRS, $P=0.430)$ nor without previous climbing $(3.5 \pm 2.7$ vs. $5.1 \pm 2.9, P=0.310)$, presyncope had an effect on pain level $(P=0.003$, estimated marginal means for pain 2.9 without pre-syncope and 8.0 with pre-syncope) in a general linear model with climbing, pre-syncope and its interaction term as fixed factors and subject as random factor. Numbness was reported in $18(45 \%)$ and palsy of the lower legs in $12(30 \%)$ cases. Clinically, marked cyanosis of the lower legs was observed during the suspension phase.

\section{Results of the linear mixed models (LMM)}

The analysis of the factors (i.e., time, climbing, pre-syncope, pain and interactions of time with climbing, time with presyncope and time with pain) that influenced the progresses of the diameter of the SFV, $\mathrm{StO}_{2}, \mathrm{HR}$, systolic BP, SV, LVEDD, LVESD and $\mathrm{ScO}_{2}$ during the suspension and postsuspension phases is presented in Table 2 .
Table $2 P$ values for fixed effects considered in the linear mixed model

\begin{tabular}{lrrrrrrrr}
\hline Fixed effect & \multicolumn{7}{l}{ Dependent variable of linear mixed model } \\
\cline { 2 - 9 } & $\mathrm{HR}$ & \multicolumn{1}{c}{$\mathrm{SBP}$} & \multicolumn{1}{c}{$\mathrm{SV}$} & \multicolumn{1}{c}{$\mathrm{StO}_{2}$} & $\mathrm{ScO}_{2}$ & $\mathrm{SFV}$ & LVEDD & LVESD \\
\hline Intercept & $<0.001$ & $<0.001$ & $<0.001$ & $<0.001$ & $<0.001$ & $<0.001$ & $<0.001$ & $<0.001$ \\
Time & $<0.001$ & $<0.001$ & $<0.001$ & $<0.001$ & 0.004 & $<0.001$ & 0.001 & 0.047 \\
Climbing & $<0.001$ & $<0.001$ & 0.648 & 0.828 & 0.607 & 0.581 & 0.109 & 0.678 \\
Pre-syncope & 0.371 & 0.036 & 0.457 & 0.985 & 0.256 & 0.035 & 0.024 & 0.042 \\
Pain & 0.603 & 0.389 & 0.694 & 0.967 & 0.387 & 0.173 & 0.019 & 0.037 \\
Climbing $\times$ time & $<0.001$ & 0.034 & 0.535 & 0.043 & 0.052 & 0.708 & 0.475 & 0.947 \\
Pre-syncope $\times$ time & 0.007 & $<0.001$ & 0.111 & 0.487 & 0.071 & 0.224 & 0.548 & 0.202 \\
Pain $\times$ time & 0.256 & 0.250 & 0.696 & 0.943 & 0.884 & 0.250 & 0.379 & 0.214 \\
\hline
\end{tabular}

$H R$ heart rate, $\mathrm{SBP}$ systolic blood pressure, $\mathrm{SV}$ stroke volume, $\mathrm{StO}_{2}$ tissue oxygen saturation, $\mathrm{ScO}$ cerebral oxygen saturation, $S F V$ diameter of the superficial femoral vein, $L V E D D$ left ventricular end-diastolic diameter, LVESD left ventricular end-systolic diameter

${ }^{a}$ For all models the covariance structure for the residuals is AR(1), except for SBP and LVEDD compound symmetry 
Time had an effect on all the parameters, while climbing (considering also its interaction with time) only on HR, systolic $\mathrm{BP}$ and $\mathrm{StO}_{2}$. For $\mathrm{ScO}_{2}$ the interaction of climbing with time did not reach statistical significance but was very close to it $(P=0.052)$. Interaction of pre-syncope with time had an effect on HR and SBP, indicating that participants with and without pre-syncope showed different progresses. Presyncope alone had an effect on SFV, LVEDD, and LVESD, indicating that participants with and without pre-syncope had a steady difference during the tests, i.e., participants who experienced a pre-syncope had a smaller diameter of the SFV $(1.1 \mathrm{~mm})$ and slightly larger ventricular diameters (1.2 $\mathrm{mm}$ for LVEDD and $1.9 \mathrm{~mm}$ for LVESD).

In the following paragraphs, for each parameter the results of the pairwise comparisons of the time points are described.

\section{Venous pooling}

\section{Diameter of the superficial femoral vein}

The mean baseline diameter of the right SFV in the supine position was $6.4 \pm 1.6 \mathrm{~mm}$ and increased to $9.9 \pm 1.8 \mathrm{~mm}$ while standing $(P<0.001)$. The mean diameter of the SFV at the beginning of the suspension phase was $10.2 \pm 2.1 \mathrm{~mm}$. During the suspension phase, the diameter did not change significantly, neither in the pre-syncope cases nor in the cases without pre-syncope. Likewise, no influence of climbing on the SFV diameter was found. At $5 \mathrm{~min}$ of the postsuspension phase (in the supine position), a decrease in the diameter of the SFV was observed $(10.6 \pm 2 \mathrm{~mm}$ at the last measurement during suspension vs. $6.2 \pm 1.6 \mathrm{~mm}$ at $5 \mathrm{~min}$ in the post-suspension phase, $P<0.001)$. SFV diameter changes during the tests are depicted in Fig. 3.

\section{Tissue oxygen saturation $\left(\mathrm{StO}_{2}\right)$ of the calf muscle}

Baseline $\mathrm{StO}_{2}$ decreased from supine to the standing position ( $70.1 \pm 6.6$ vs. $56.4 \pm 5.6 \%, P<0.001)$. During the first $5 \mathrm{~min}$ of the suspension phase, a decrease in $\mathrm{StO}_{2}$ was detected in participants who did not climb (57.3 \pm 5.5 vs. $52.4 \pm 6.1 \%$, $P=0.001$ ), while $\mathrm{StO}_{2}$ did not change when participants had climbed before suspension $(53.2 \pm 4.4$ vs. $52.8 \pm 4.6 \%$, $P=1.000$ ). From minute 5 of the suspension phase to $3 \mathrm{~min}$ before end of the suspension phase, $\mathrm{StO}_{2}$ decreased further in both groups $(52.6 \pm 5.3$ vs. $48.7 \pm 5.6 \%, P=0.002)$. During the first $5 \mathrm{~min}$ of the post-suspension phase, an increase in $\mathrm{StO}_{2}$ was observed $(49.4 \pm 6.5$ vs. $63.6 \pm 5.1 \%, P<0.001)$. $\mathrm{StO}_{2}$ changes during the tests are depicted in Fig. 3.

\section{Localized bioelectrical-impedance analysis}

The resistance $(R)(200.2 \pm 18.6$ vs. $193.6 \pm 17.9, P<0.001)$ and reactance $(\mathrm{Xc})(27.7 \pm 4.2$ vs. $26.6 \pm 3.7, P=0.001)$ changed from supine to standing position. Xc further decreased from the start to the last measure during the suspension phase $(26.4 \pm 3.6$ vs. $22.9 \pm 3.1, P<0.001)$ while $R$ $(192.3 \pm 18.3$ vs. $190.7 \pm 17.6, P=0.201)$ did not change. The general linear model showed no effect of climbing ( $P=0.514$ and $P=0.253$ for $R$ and $\mathrm{Xc}$, respectively) or pre-syncope ( $P=0.981$ and $P=0.701$ for $R$ and Xc, respectively) from the start to the last measure during the suspension phase.

\section{Hemodynamic changes}

\section{Heart rate}

Baseline HR increased from supine to the standing position $(63.1 \pm 7.9$ vs. $72.4 \pm 9.4 \mathrm{bpm}, P<0.001)$. During suspension, HR decreased from the start to 3 min before the end of the suspension phase in participants who had climbed before $(113.3 \pm 15.9$ vs. $96.0 \pm 18.5 \mathrm{bpm}, P<0.001)$ but did not change in participants who had not climbed $(79.8 \pm 14.4$ vs. $84.4 \pm 15.2 \mathrm{bpm}$, respectively, $P=0.098$ ). In participants with pre-syncope, HR decreased in the last $3 \mathrm{~min}$ before the pre-syncopal event $(96.3 \pm 22.4$ vs. $82.2 \pm 19.4 \mathrm{bpm}$, $P=0.043$ ) and decreased further during the first $5 \mathrm{~min}$ of the post-suspension phase $(82.2 \pm 19.4$ vs. $62.9 \pm 13.5 \mathrm{bpm}$, $P=0.033$ ). In participants who did not manifest a pre-syncope, HR remained stable until the end of the suspension phase and decreased only in the first 5 min of the post-suspension phase ( $86.4 \pm 15.8$ vs. $60.3 \pm 10.1 \mathrm{bpm}, P<0.001)$. HR changes are depicted in Fig. 4.

\section{Systolic blood pressure}

Systolic BP increased at baseline from supine to standing $(121.1 \pm 13.2$ vs. $138.7 \pm 14.7 \mathrm{mmHg}, P<0.001)$. In participants who had not climbed before the test, systolic BP did not change from the start of the suspension phase until $3 \mathrm{~min}$ before end of the suspension phase $(143.8 \pm 12.8$ vs. $148.0 \pm 15.3 \mathrm{mmHg}, P=0.272)$ but increased in participants who had climbed before $(123.6 \pm 17.1$ vs. $139.2 \pm 21.8 \mathrm{mmHg}, P=0.034)$. In participants with presyncope, systolic BP decreased significantly in the last $3 \mathrm{~min}$ before the pre-syncopal event $(142.5 \pm 18.6$ vs. $101.6 \pm 17.4 \mathrm{mmHg}, P=0.001)$ while it did not change in participants without a pre-syncope $(144.1 \pm 19.6$ vs. $141.7 \pm 20.4 \mathrm{mmHg}, P=$ n.s.). During the first $5 \mathrm{~min}$ of the post-suspension phase, systolic BP decreased in participants 


\section{Diameter SFV}

No pre-syncope
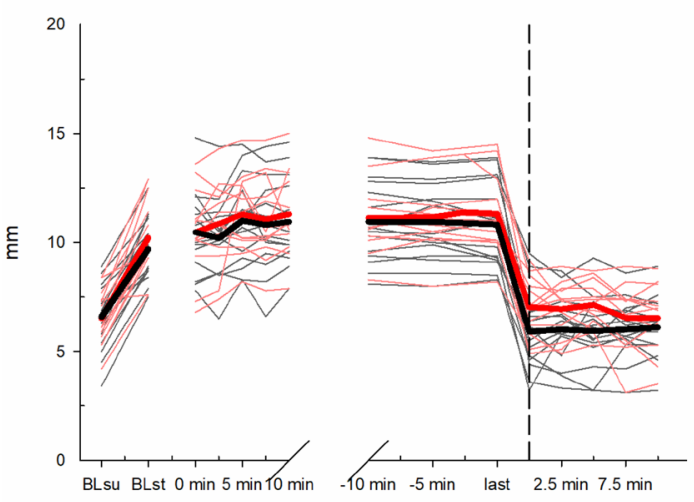

No pre-syncope
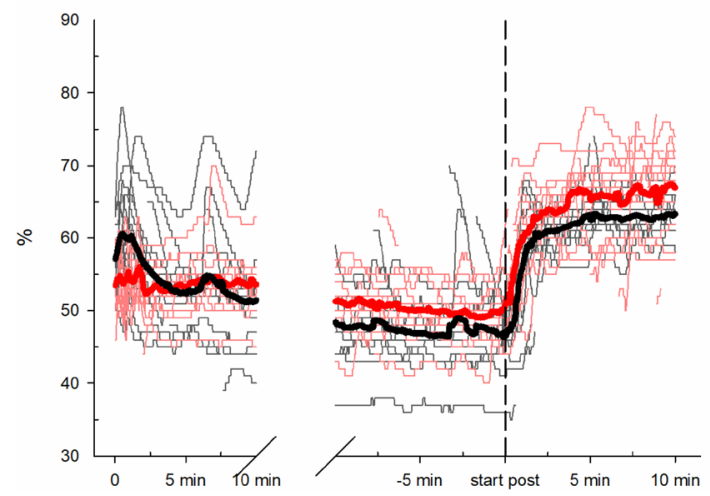

Fig. 3 Progress of $\mathrm{StO}_{2}$ and SFV during suspension test. Black colour represents progresses without climbing before the test and red colour represents progresses with previous climbing. The two thicker lines represent the mean values of the two groups and the black vertical

without a pre-syncope $(141.7 \pm 20.4$ vs. $128.9 \pm 17.8 \mathrm{mmHg}$, $P=0.038$ ) and increased in participants who had a presyncope (though not reaching statistical significance; $101.6 \pm 17.4$ vs. $124.9 \pm 18.2 \mathrm{mmHg}, P=$ n.s.). Systolic BP changes are depicted in Fig. 4.

\section{Stroke volume}

In both participants with and without climbing before the suspension phase, stroke volume decreased from the beginning of the suspension phase to $3 \mathrm{~min}$ before the end of the suspension $(80.1 \pm 14.7$ vs. $69.7 \pm 15.9 \mathrm{ml}$,
Pre-syncope

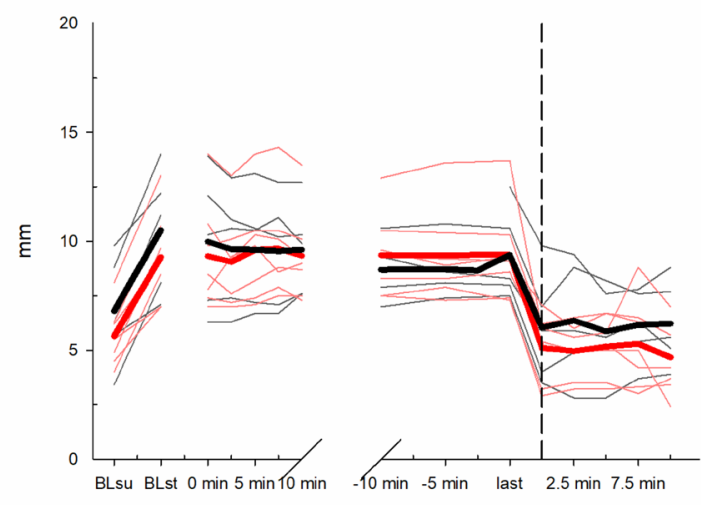

$\mathrm{StO}_{2}$

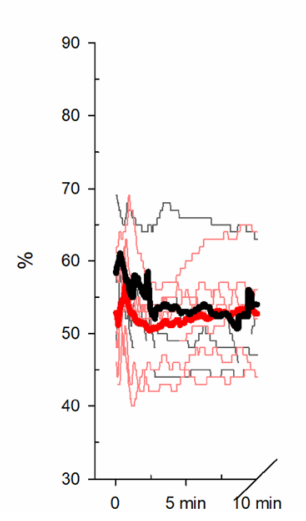

Pre-syncope

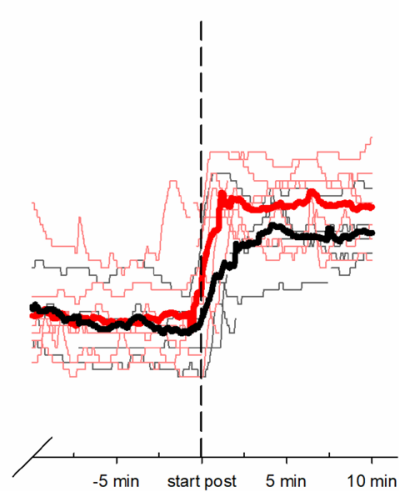

dashed line at the start of post-hanging phase. BLsu baseline value in supine position, BLst baseline value in standing position, last last measure before end of the test

$P=0.007)$. No statistically significant reduction of stroke volume in participants with a pre-syncope was detected before the occurrence of signs and symptoms of pre-syncope. In the first $5 \mathrm{~min}$ of the post-suspension phase, SV increased $(73.5 \pm 16.5$ vs. $98.9 \pm 14.9 \mathrm{ml}, P<0.001)$. SV changes are depicted in Fig. 4.

\section{Left ventricular diameters}

LVEDD Baseline LVEDD decreased from supine to standing $(44.0 \pm 4.2$ vs. $40.7 \pm 3.8 \mathrm{~mm}, P<0.001)$. During the suspension phase, no significant change in LVEDD was 
Fig. 4 Progress of HR, SBP, SV and LVD during suspension test. Black colour represents progresses without climbing before the test and red colour represents progresses with previous climbing. The two thicker lines represent the mean values of the two groups and the black vertical dashed line at the start of post-hanging phase. $B L s u$ baseline value in supine position, $B L s t$ baseline value in standing position, last last measure before end of the test
No pre-syncope

HR
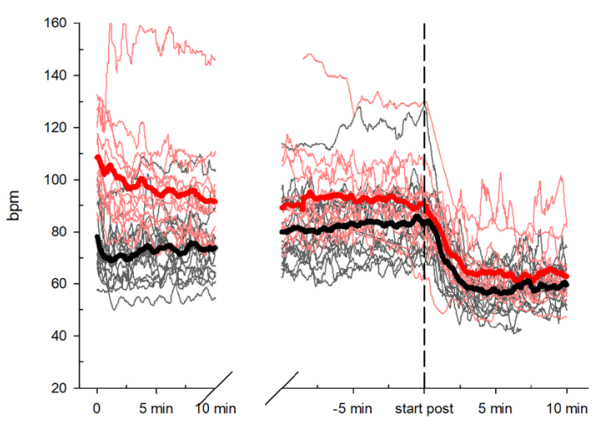

No pre-syncope

SBP
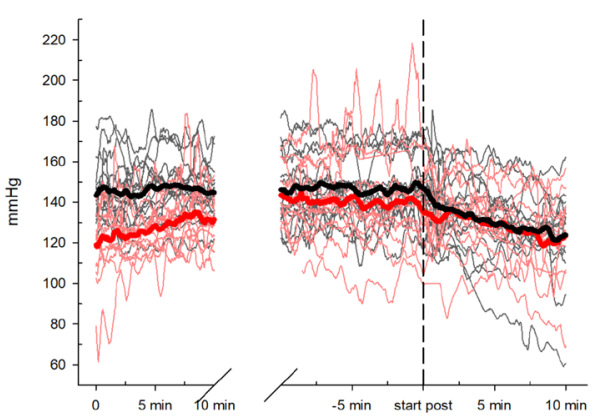

No pre-syncope
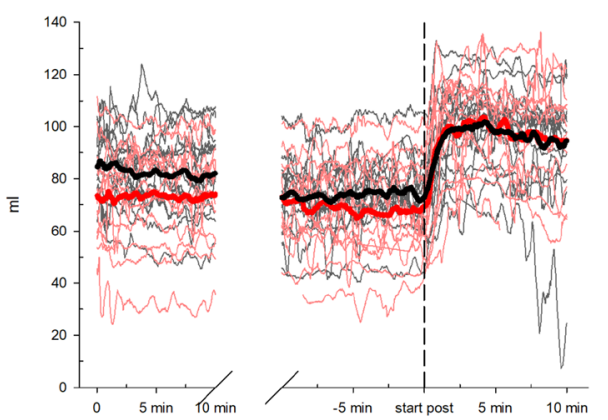

SV
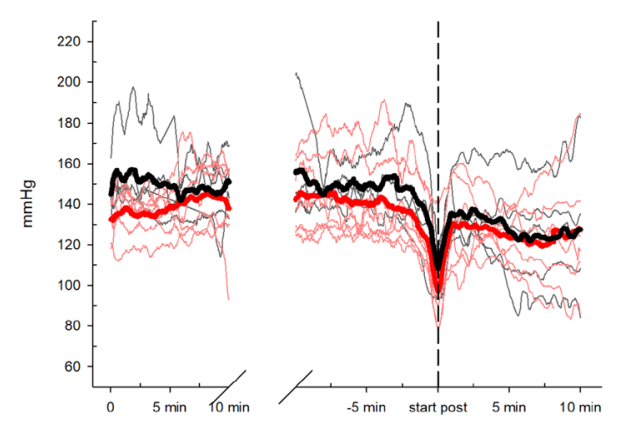

LVEDD
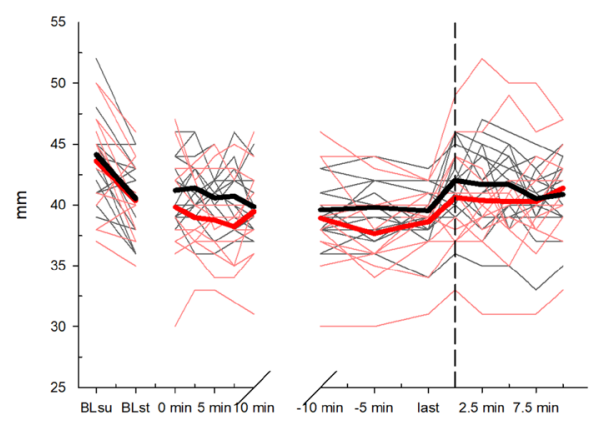

Pre-syncope
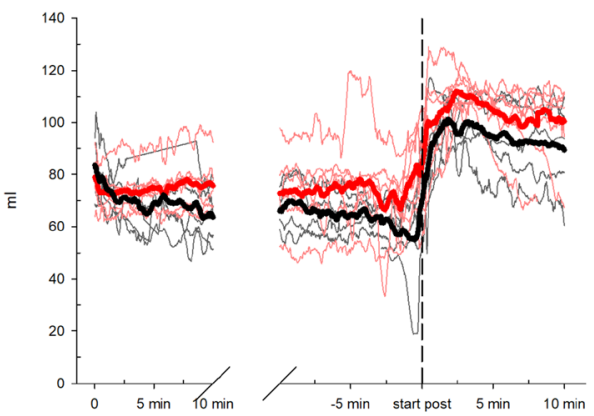

Pre-syncope

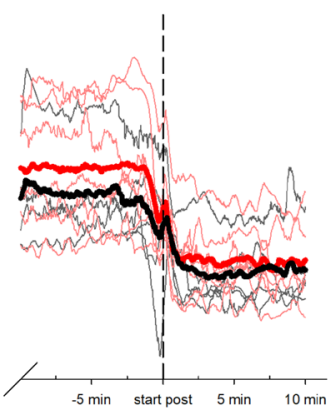

Pre-syncope

Pre-syncope
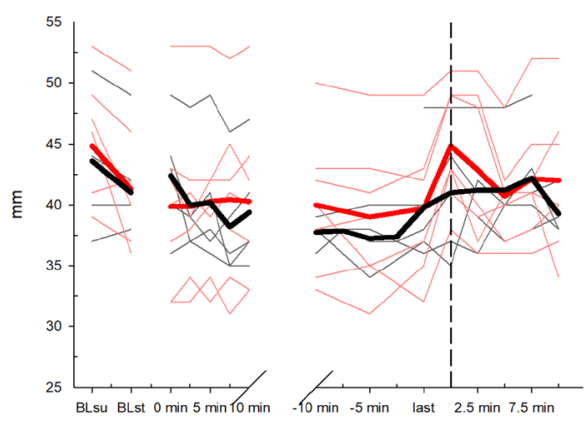
observed. LVEDD increased from the last measure taken before the end of suspension to the start of post-suspension phase $(39.3 \pm 3.6 \mathrm{~mm}$ vs. $41.9 \pm 4.2 \mathrm{~mm}, P=0.001)$. LVEDD changes are depicted in Fig. 4.

LVESD LVESD did not change at baseline from supine to standing ( $27.6 \pm 3.9 \mathrm{~mm}$ vs. $26.5 \pm 3.6 \mathrm{~mm}, P=0.098)$. No pairwise comparison of the time points was significant.

\section{Cerebral oxygen saturation $\left(\mathrm{ScO}_{2}\right)$}

$\mathrm{ScO}_{2}$ did not change from supine to standing at baseline $(67.9 \pm 4.7$ vs. $67.2 \pm 4.3 \%, P=0.199)$. Between the start and 3 min before the end of suspension phase, a statistically significant increase in $\mathrm{ScO}_{2}$ was observed in participants who did not climb before suspension $(66.3 \pm 4.5$ vs. $69.9 \pm 5.6 \%$, $P=0.001$; relative change from start $+5.4 \% \pm 5.2 \%$ ), whereas in those with prior climbing, $\mathrm{ScO}_{2}$ remained constant $(70.3 \pm 5.8$ vs. $69.8 \pm 5.9 \%, P=$ n.s.; relative change from start $-0.5 \% \pm 7.1 \%$ ). In the participants with pre-syncope, $\mathrm{ScO}_{2}$ did not change significantly in the 3 min preceding the pre-syncope $(73.0 \pm 6.2$ vs. $69.1 \pm 6.2 \%, P=$ n.s.; relative changes from start $+3.8 \% \pm 8.6 \%$ vs. $-1.8 \% \pm 7.1 \%$ ). Also during the first $5 \mathrm{~min}$ of the post-suspension phase, $\mathrm{ScO}_{2}$ did not change $(68.4 \pm 5.5$ vs. $69.5 \pm 5.2 \%, P=0.194$; relative changes from start $+0.4 \% \pm 6.8 \%$ vs. $+2.0 \% \pm 7.1 \%$ ).

\section{Baroreceptor-sensitivity}

39 out of 40 tests were included in the baroreceptor-sensitivity analysis (one with missing data was excluded). Overall, no time effect of pre-syncope on baroreceptor-sensitivity was found $(P=0.308)$. Nonetheless, in the first minutes during suspension in the tests without prior climbing, baroreceptorsensitivity was higher in participants without syncope compared to the ones with pre-syncope (12.6 \pm 3.6 vs. $7.7 \pm 3.8$, $P=0.026)$. In the tests with prior climbing, no such differences were found ( $7.2 \pm 3.3$ vs. $6.7 \pm 3.5, P=0.782)$.

\section{Discussion}

In our study, we observed a pre-syncope during suspension in a sit harness in $30 \%$ of all tests. Time to pre-syncope was highly variable and unpredictable with a sudden occurrence of signs and symptoms without a prodromal stage. Venous pooling in the lower leg could be evidenced very early in all tests; however, the reduced preload did not seem to have any relevant impact on macro-hemodynamics. In participants with a pre-syncope, a sudden decrease in HR and systolic BP was observed, resembling a vagal mechanism.

The principal aim of our study was to clarify the pathophysiology of suspension syndrome and particularly to differentiate between an important reduction in cardiac preload due to venous pooling, and a vagally mediated circulatory collapse. A hemodynamically relevant venous pooling, causing a reduction in cardiac preload, would be expected to elicit compensatory mechanisms to preserve cardiac output, such as progressive tachycardia. Moreover, a decrease in LVEDD and LVESD and finally a drop of BP would be anticipated (Pacagnella et al. 2013). In contrast, a vagal event would be associated with vasodepressor hypotension and/or inappropriate bradycardia (Kapoor 2000; Shen et al. 2017).

To measure venous pooling in the lower leg, we used the diameter of the SFV, the $\mathrm{StO}_{2}$ of the calf muscle and the localized bioelectrical-impedance. A shift of blood to dependent parts of the body leads to filling of capacitance vessels in the leg with an increase in diameter (Marshall et al. 2014). Using ultrasound, the increase in diameter of large veins, such as the SFV, can easily be monitored over time. However, measurement of the orthostatic blood volume accumulation in the small vessels is more challenging. To track intravascular orthostatic volume changes within the small blood vessels of the lower leg, a new four-wavelength generation NIRS monitor has been used (Ferraris et al. 2018). NIRS tissue oxygen saturation measurement is made on a mixture of both venous and arterial blood with a mean ratio of this mixture estimated to be $70 \%$ venous $-30 \%$ arterial (Benni et al. 2018). A decrease in $\mathrm{StO}_{2}$ thus indicates either accumulation of de-saturated venous blood or a reduced oxygen delivery (Bartok and Schoeller 2004; Binzoni et al. 2000, 2003; Habazettl et al. 2016; Hachiya et al. 2004; Truijen et al. 2012). The observed decrease in $\mathrm{StO}_{2}$ during suspension was attributed to an accumulation of venous blood rather than a reduced oxygen supply, as no compression or flow limitation of the arterial vessels (e.g., by the harness straps) was observed with ultrasound and stroke volume and arterial oxygen saturation were constant. As a third method to monitor regional circulation and fluid accumulation in the calf muscle-localized bioelectricalimpedance components have been utilized (Lukaski 2013). Our study is the first to quantify venous pooling during suspension using these three distinct modalities. We found that immediately after the beginning of the suspension phase, a marked and rapid accumulation of venous blood in the superficial femoral vein occurred. That is, the diameter of the SFV immediately increased to values comparable to those measured at baseline after 2 min of upright standing. However, during the suspension phase, the diameter of the SFV did not progressively increase, supposedly because the maximum distending capacity of the venous vessel had been already reached. Concomitantly to the initial increase in SFV diameter, a remarkable decrease in $\mathrm{StO}_{2}$ measured with NIRS was observed. Most likely, this can be explained by an accumulation of desaturated blood in venules (Habazettl et al. 2016), 
which was in line with the observation of a marked cyanosis of the feet, observed in nearly all participants. After the initial pronounced drop of the $\mathrm{StO}_{2}$, a steady but more gradual decrease was observed. Together with the constant diameter of the SFV, this finding indicates progressive venous pooling at the level of small venous vessels. Similar to the SFV diameter, $R$ values during suspension were comparable to the values attained at baseline during standing and were lower than the values recorded in the supine position, which might be a consequence of a gravitationally induced blood pooling in abdomen and legs. Interestingly, whereas $R$ remained stable in the course of the suspension, which indicates no additional fluid volume increase, Xc further decreased. This might indicate fluid shifts between extracellular and intracellular compartments (Gatterer et al. 2014) and/or changes in blood circulation properties (Varlet-Marie et al. 2016), which could have occurred in the course of the suspension. Yet, the exact meaning of the changed Xc cannot be explained by this study and at present this interpretation has to be viewed with caution.

The accumulation of blood in the dependent body regions, however, seemed not to have any substantial impact on macro-hemodynamics, which we assessed by continuous measurement of HR, BP and SV and intermittent quantification of LVEDD and LVESD. We found that, despite venous pooling, only SV slightly decreased during suspension. Despite the reduction in SV, blood pressure remained constant because of a compensatory increase in vascular resistance. Importantly, venous pooling did not elicit a compensatory increase in HR, which would definitely be expected if the preload was reduced to a significant degree, similarly as seen in hypovolemic/hemorrhagic shock (Salomao et al. 2015). Furthermore, LVEDD did not decrease, and, taken together, our findings contradict the hypothesis of a marked preload reduction leading to an "empty beating heart". Quite in contrast, all pre-syncope cases in our study followed a uniform pattern, favoring the hypothesis of a vagal event. Indeed, the pre-syncope was characterized by a pronounced decrease in systolic BP and HR as in a vasovagal syncope with a mixed vasodepressive and cardioinhibitory pattern. The haemodynamic findings were preceded by a sudden occurrence of dizziness, light-headedness, pale skin, warmth, blurred vision or nausea, lasting from a few seconds to a few minutes. The haemodynamic pattern observed in our study is identical to that found in patients with a vasovagal syncope in the tilt table test (Brignole et al. 2000; Gaggioli et al. 2000). Thus, we hypothesize that the susceptibility of an individual to develop suspension syndrome could be predicted in a tilt test. However, this needs to be proven in a future trial.

The mechanism behind a vagal syncope has not been totally elucidated (Mosqueda-Garcia et al. 2000). One hypothesis implies the ventricular muscle to contract on a nearly empty chamber, which, in turn, is thought to stimulate afferent signals from the left ventricle to the brainstem, inhibiting sympathetic outflow to blood vessels and the heart, resulting in arterial hypotension and bradycardia (Bezold-Jarisch reflex) (Mark 1983). Our findings do not corroborate this theory, as referred to above. Another hypothesis involves a dysfunction of the arterial baroreceptor reflex. The baroreceptor reflex is a primary homeostatic mechanism that maintains arterial blood pressure within narrow limits via changes in cardiac output and systemic vascular resistance, mediated by the autonomic nervous system. Several authors have advocated defective baroreflex function as a potential mechanism accounting for the development of vasovagal syncope (Ellenbogen et al. 1997; MosquedaGarcia et al. 1997). In our study, participants experiencing a pre-syncope showed lower baroreceptor-sensitivity values during the initial phase of suspension (only visible in the trial without prior climbing), though, baroreceptor-sensitivity was unchanged during suspension. Particularly, no change in baroreceptor-sensitivity was found in the minutes preceding the pre-syncope. This neither confirms nor refutes the hypothesis of a baroreflex dysfunction in the pathophysiology of vagal syncope.

Pain is a known trigger for vagally mediated syncopal events (Hainsworth 2003) and in our study, 75\% of participants who experienced a pre-syncope reported significant pain ( $\geq 3$ on a NRS). By means of a general linear model, we found that pain level differed between participants with and without pre-syncope, thus we can conclude that pain seems to be an important factor contributing to the vagal event.

The time to pre-syncope was variable and unpredictable and the signs and symptoms of pre-syncope occurred suddenly without a prodromal stage. The shortest time to presyncope in our study was 13.4 min with a mean time to pre-syncope of $44.7 \mathrm{~min}$ and a maximal time of $59 \mathrm{~min}$, respectively. This is comparable to a recent study on suspension syndrome, which found a mean time to syncope of 30 min with a range from 20 to 36 min (Lanfranconi et al. 2017). Four volunteers from our study experienced a presyncope during both tests, with and without prior climbing, and two of them had a history of a past vagal syncope. This supports the hypothesis that, though the vagal response is present in all healthy humans, inter-individual differences in susceptibility seem to exist (Alboni et al. 2007) and persons prone to vagally mediated syncopal events might be more susceptible to suspension syndrome.

Similarly to a post-exercise syncope (Lowe and Petch 2000; O'Connor et al. 1999; Shen et al. 2017), we assumed that climbing before suspension would increase the susceptibility and decrease the time until the occurrence of suspension syndrome, particularly if a vagal mechanism was involved. In the four participants who experienced a pre-syncope in both tests, the mean time to pre-syncope was shorter 


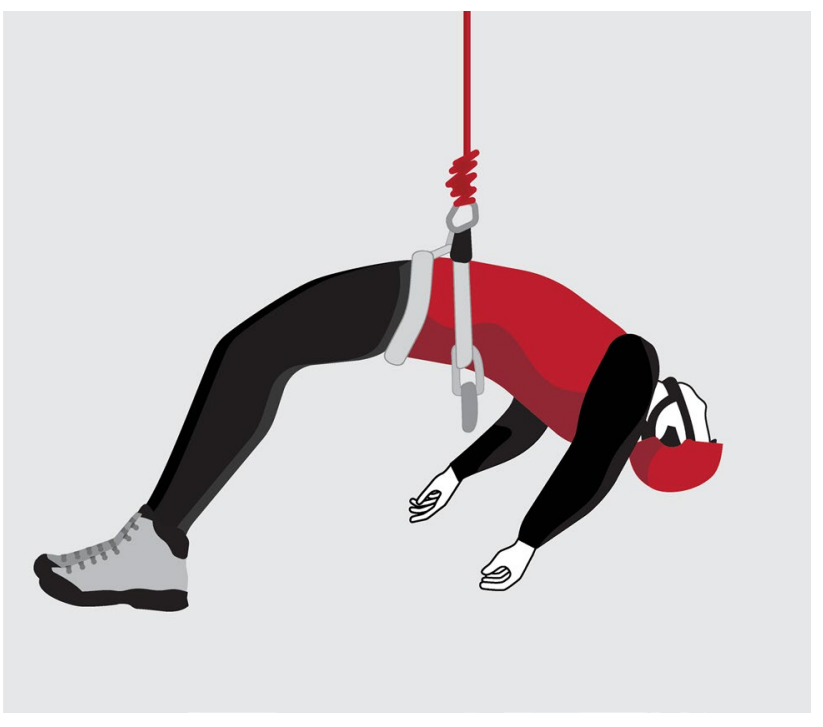

Fig. 5 In an unconscious person suspended in a sit harness, the attachment point of the rope, located at the level of the hips will represent the highest point

when they climbed before suspension and of the remaining four pre-syncopal cases, three occurred after climbing, supporting our hypothesis.

We showed that time until loss of consciousness while suspended on a rope is unpredictable. It is therefore recommended, that persons suspended on a rope and not able to free themselves should be rescued as soon as possible, not least because loss of consciousness while hanging on a rope can rapidly become life threatening: While the syncopal fall normally will accomplish a horizontal position, allowing a rapid restoration of cerebral perfusion and rapid recovery (Weimer and Zadeh 2009), this is not the case when hanging vertically on a rope. In an unconscious person suspended in a sit harness, the attachment point of the rope, located at the level of the hips will represent the highest point (Fig. 5), impeding the return flow of blood accumulated in the veins of the lower legs. Thus, perfusion of the vital organs may not be restored and the person may eventually die.

Until today, it is unclear how to position a casualty appropriately once rescued from suspension. Although most authors recommend a supine position (Adisesh et al. 2011; Thomassen et al. 2009) according to the advanced life support guidelines (Soar et al. 2015), some still advise a semi-recumbent position first, with the idea to avoid an acute ventricular volume overload. In our study, we decided to bring participants with a pre-syncope in a supine position. As expected, LVEDD increased in the horizontal position, however, the diameter did not even reach the supine baseline value, contradicting the hypothesis of a ventricular overdistension. Moreover, all participants with a pre-syncope recovered within seconds in the supine position. Therefore, we recommend that persons hanging on a rope and showing signs of a suspension syndrome should be rescued and put into supine position as soon as possible.

\section{Limitations}

Participants were asked to move their legs as little as possible while suspended, to facilitate the measurements of venous pooling (particularly ultrasonografic measurements). The reduced leg movements might have influenced the degree of venous pooling and the time to pre-syncope. In real life, persons hanging freely on a rope will probably move their legs spontaneously, possibly reducing venous pooling, and delaying loss of consciousness. However, syncope will remain a sudden event and exhaustion, injuries, hypoglycemia, hypothermia, pain etc. might preclude antigravity muscle activity when being suspended on a rope outdoors.

We used echocardiography to monitor left ventricular dimensions as a measure of ventricular filling. Ventricular diameter is not an ideal parameter for preload and only a considerable ventricular underfilling could have been detected. Moreover, assessment of right ventricular dimensions might have been more appropriate to exclude ventricular volume overload once the participants have been brought in a supine position. However, especially during the suspension phase, the acoustic window was not ideal to depict the right side of the heart. Though the dimensions of the right ventricle were not assessed directly, no other signs of right ventricular volume overload, such as a diastolic septal displacement, were apparent. Even though localized bioimpedance analysis has been shown to identify local fluid accumulation (Nescolarde et al. 2015) and to be related to blood rheology (Varlet-Marie et al. 2016), the method is not well validated yet and thus these results have to be interpreted with caution.

\section{Conclusions}

We found a surprisingly high rate $(30 \%)$ of pre-syncopal episodes in our study and in all cases, a uniform circulatory and hemodynamic pattern could be observed: Blood pools in the veins of the lower legs, however, no relevant impact on macro-hemodynamics was detected. Pre-syncope was characterized by a sudden decrease in BP and $\mathrm{HR}$, indicating a vagally driven mechanism with a mixed vasodepressive and cardioinhibitory pattern; pain during suspension may act as a trigger to evoke the reflex. Time to pre-syncope is unpredictable and persons suspended on 
a rope should be rescued and put into a supine position as soon as possible.

Acknowledgements We thank the Mountain Rescue South Tyrol (Bergrettungsdienst im Alpenverein Südtirol) for putting their indoor climbing facility at our disposal. Furthermore, we thank the companies Mindray Bio-Medical Electronics Co., Ltd., Masimo Corporation, Akern and Salewa for lending the equipment. We would like to acknowledge the support of Prof. Luciano Bernardi in interpreting the data on baroreflex sensitivity.

Author contributions SR, PP, KS, GS, HG and HB conceived and designed research. SR, KS, GS, MP, ME, LO and BB conducted experiments. SR, TdC, KS and HG analyzed data. SR, TdC, HG and PP wrote the manuscript. All authors read and approved the manuscript.

Funding The study was funded by a research Grant of the German Society for Mountain and Expedition Medicine.

Open Access This article is distributed under the terms of the Creative Commons Attribution 4.0 International License (http://creativeco mmons.org/licenses/by/4.0/), which permits unrestricted use, distribution, and reproduction in any medium, provided you give appropriate credit to the original author(s) and the source, provide a link to the Creative Commons license, and indicate if changes were made.

\section{References}

Adisesh A, Lee C, Porter K (2011) Harness suspension and first aid management: development of an evidence-based guideline. Emerg Med J 28:265-268. https://doi.org/10.1136/emj.2010.097246

Alboni P, Brignole M, Degli Uberti EC (2007) Is vasovagal syncope a disease? Europace 9:83-87. https://doi.org/10.1093/europace/ eul179

Bartok C, Schoeller DA (2004) Estimation of segmental muscle volume by bioelectrical impedance spectroscopy. J Appl Physiol (Bethesda Md 1985) 96:161-166. https://doi.org/10.1152/jappl physiol.00686.2002

Benni PB, MacLeod D, Ikeda K, Lin H-M (2018) A validation method for near-infrared spectroscopy based tissue oximeters for cerebral and somatic tissue oxygen saturation measurements. J Clin Monit Comput 32:269-284. https://doi.org/10.1007/s10877-017-0015-1

Bernardi L, De Barbieri G, Rosengard-Barlund M, Makinen VP, Porta C, Groop PH (2010) New method to measure and improve consistency of baroreflex sensitivity values. Clin Auton Res 20:353-361. https://doi.org/10.1007/s10286-010-0079-1

Binzoni T, Quaresima V, Ferrari M, Hiltbrand E, Cerretelli P (2000) Human calf microvascular compliance measured by near-infrared spectroscopy. J Appl Physiol (Bethesda Md 1985) 88:369-372. https://doi.org/10.1152/jappl.2000.88.2.369

Binzoni T, Ngo L, Girardis M, Springett R, Terrier F, Delpy D (2003) Venous-arteriolar reflex in human gastrocnemius studied by NIRS Adv. Exp Med Biol 530:467-473

Breivik H et al (2008) Assessment of pain BJA. Br J Anaesth 101:1724. https://doi.org/10.1093/bja/aen103

Brignole M et al (2000) New classification of haemodynamics of vasovagal syncope: beyond the VASIS classification. Analysis of the pre-syncopal phase of the tilt test without and with nitroglycerin challenge. Vasovagal Syncope Int Study Eur 2:66-76

Council for International Organizations of Medical Sciences (2002) International ethical guidelines for biomedical research involving human subjects. Bull Med Ethics 182:17-23
Council of Europe (1997) Convention for protection of human rights and dignity of the human being with regard to the application of biology and biomedicine: convention of human rights and biomedicine. Kennedy Inst Ethics J 7:277-290

Ellenbogen KA, Morillo CA, Wood MA, Gilligan DM, Eckberg DL, Smith ML (1997) Neural monitoring of vasovagal syncope. Pacing Clin Electrophysiol 20:788-794

Ferraris A, Jacquet-Lagreze M, Fellahi JL (2018) Four-wavelength near-infrared peripheral oximetry in cardiac surgery patients: a comparison between EQUANOX and O3. J Clin Monit Comput 32:253-259. https://doi.org/10.1007/s10877-017-0025-z

Flora G (1972a) Fatal and non-fatal accidents involving falls into the rope. Paper presented at the 2 nd international conference of mountain rescue doctors, Innsbruck (Austria), November 1972

Flora G (1972b) Fatal and non-fatal accidents involving falls into the rope. Paper presented at the 2nd international conference of mountain rescue doctors, Innsbruck (Austria)

Gaggioli G et al (2000) New classification of haemodynamics of vasovagal syncope: beyond the VASIS classification: analysis of the pre-syncopal phase of the tilt test without and with nitroglycerin challenge. EP Eur 2:66-76. https://doi.org/10.1053/ eupc. 1999.0064

Gatterer H, Schenk K, Laninschegg L, Schlemmer P, Lukaski H, Burtscher M (2014) Bioimpedance identifies body fluid loss after exercise in the heat: a pilot study with body cooling. PloS One 9:e109729. https://doi.org/10.1371/journal.pone.0109729

Habazettl H, Stahn A, Nitsche A, Nordine M, Pries AR, Gunga HC, Opatz O (2016) Microvascular responses to (hyper-)gravitational stress by short-arm human centrifuge: arteriolar vasoconstriction and venous pooling. Eur J Appl Physiol 116:57-65. https://doi. org/10.1007/s00421-015-3241-6

Hachiya T, Blaber AP, Saito M (2004) Changes in superficial blood distribution in thigh muscle during LBNP assessed by NIRS. Aviat Space Environ Med 75:118-122

Hainsworth R (2003) Syncope: what is the trigger? Heart 89:123-124

Halliwill JR, Sieck DC, Romero SA, Buck TM, Ely MR (2014) Blood pressure regulation $\mathrm{X}$ : what happens when the muscle pump is lost? Post-exercise hypotension and syncope. Eur J Appl Physiol 114:561-578. https://doi.org/10.1007/s00421-013-2761-1

Kapoor WN (2000) Syncope. N Engl J Med 343:1856-1862. https:// doi.org/10.1056/nejm200012213432507

Lanfranconi F, Pollastri L, Corna G, Bartesaghi M, Novarina M, Ferri A, Miserocchi GA (2017) The elusive path of brain tissue oxygenation and cerebral perfusion in harness hang syncope in mountain climbers high. Alt Med Biol 18:363-371. https://doi.org/10.1089/ ham.2017.0028

Lee C, Porter KM (2007) Suspension trauma. Emerg Med J 24:237238. https://doi.org/10.1136/emj.2007.046391

Lowe MD, Petch MC (2000) Syncope after effort. Postgrad Med J 76:164-165. https://doi.org/10.1136/pmj.76.893.164

Lukaski HC (2013) Evolution of bioimpedance: a circuitous journey from estimation of physiological function to assessment of body composition and a return to clinical research. Eur J Clin Nutr 67(Suppl 1):S2-S9. https://doi.org/10.1038/ejen.2012.149

Mark AL (1983) The Bezold-Jarisch reflex revisited: clinical implications of inhibitory reflexes originating in the heart. J Am Coll Cardiol 1:90-102

Marshall M, Schwahn-Schreiber C, Kleine MW (2014) Patient positioning for duplex ultrasound scanning of the leg vessels. Phlebologie 43:201-205. https://doi.org/10.12687/phleb2180-4-2014

Mehta Y, Arora D (2014) Newer methods of cardiac output monitoring. World J Cardiol 6:1022-1029. https://doi.org/10.4330/wjc. v6.i9.1022

Mosqueda-Garcia R et al (1997) Sympathetic and baroreceptor reflex function in neurally mediated syncope evoked by tilt. J Clin Investig 99:2736-2744. https://doi.org/10.1172/jci119463 
Mosqueda-Garcia R, Furlan R, Tank J, Fernandez-Violante R (2000) The elusive pathophysiology of neurally mediated syncope. Circulation 102:2898-2906

Mössmer GSM (2013) Sportklettern. Sicher unterwegs in Halle und Klettergarten. ÖAV

Nescolarde L, Yanguas J, Lukaski H, Alomar X, Rosell-Ferrer J, Rodas G (2015) Effects of muscle injury severity on localized bioimpedance measurements. Physiol Meas 36:27-42. https://doi. org/10.1088/0967-3334/36/1/27

O'Connor FG, Oriscello RG, Levine BD (1999) Exercise-related syncope in the young athlete: reassurance, restriction or referral? Am Fam Physician 60:2001-2008

Pacagnella RC, Souza JP, Durocher J, Perel P, Blum J, Winikoff B, Gulmezoglu AM (2013) A systematic review of the relationship between blood loss and clinical signs. PLoS One 8:e57594. https ://doi.org/10.1371/journal.pone.0057594

Pasquier M, Yersin B, Vallotton L, Carron PN (2011) Clinical update: suspension trauma. Wilderness Environ Med 22:167-171. https ://doi.org/10.1016/j.wem.2010.12.006

Roeggla M et al (1996) Cardiorespiratory response to free suspension simulating the situation between fall and rescue in a rock climbing accident. Wilderness Environ Med 7:109-114

Roggla G, Moser B, Roggla M (2008) Suspension trauma. Emerg Med J 25:59-59

Salomao E Jr, Otsuki DA, Correa AL, Fantoni DT, dos Santos F, Irigoyen MC, Auler JO Jr (2015) Heart Rate variability analysis in an experimental model of hemorrhagic shock and resuscitation in pigs. PloS One 10:e0134387. https://doi.org/10.1371/journ al.pone. 0134387

Shen W-K et al (2017) 2017 ACC/AHA/HRS guideline for the evaluation and management of patients with syncope: a report of the American College of Cardiology/American Heart Association Task Force on clinical practice guidelines, and the heart rhythm society circulation. Circulation 136(5):e60-e122. https://doi. org/10.1161/cir.0000000000000499

Soar J et al (2015) European resuscitation council guidelines for resuscitation 2015: section 3. Adult Adv Life Support Resusc 95:100$147 \mathrm{https} / / /$ doi.org/10.1016/j.resuscitation.2015.07.016

R Development Core Team (2015) R: a language and environment for statistical computing. R Foundation for Statistical Computing, Vienna

Thomassen O, Skaiaa SC, Brattebo G, Heltne JK, Dahlberg T, Sunde GA (2009) Does the horizontal position increase risk of rescue death following suspension trauma? Emerg Med J 26:896-898. https://doi.org/10.1136/emj.2008.064931

Truijen J et al (2012) Orthostatic leg blood volume changes assessed by near-infrared spectroscopy. Exp Physiol 97:353-361. https:// doi.org/10.1113/expphysiol.2011.061051

Varlet-Marie E, Jore C, Brun JF (2016) Segmental bioelectrical impedance analysis (SBIA) and blood rheology: reducing the gap between in vivo and in vitro? Clin Hemorheol Microcirc 64:603-611. https://doi.org/10.3233/ch-168041

Weems B, Bishop P (2003) Will your safety harness kill you? Occup Health Saf (Waco Tex) 72:86-88, 90

Weimer LH, Zadeh P (2009) Neurological aspects of syncope and orthostatic intolerance. Med Clin N Am 93:427-449. https://doi. org/10.1016/j.mcna.2008.10.002

World Medical Association Declaration of Helsinki (2013) Ethical principles for medical research involving human subjects. JAMA 310:2191-2194. https://doi.org/10.1001/jama.2013.281053

Publisher's Note Springer Nature remains neutral with regard to jurisdictional claims in published maps and institutional affiliations. 